\title{
VOLTERRA-TYPE OPERATORS FROM ANALYTIC MORREY SPACES TO BLOCH SPACE
}

\author{
ZHENGYUAN ZHUO AND SHANLI YE \\ Communicated by Hermann Brunner
}

\begin{abstract}
In this note, we study the boundedness and compactness of integral operators $I_{g}$ and $T_{g}$ from analytic Morrey spaces to Bloch space. Furthermore, the norm and essential norm of those operators are given.
\end{abstract}

1. Introduction. Let $\mathbb{D}=\{z:|z|<1\}$ and $\partial \mathbb{D}=\{z:|z|=1\}$ denote, respectively, the open unit disc and the unit circle in the complex plane $\mathbb{C}$. Let $H(\mathbb{D})$ be the space of all analytic functions on $\mathbb{D}$ and $d m(z)=\frac{1}{\pi} d x d y$ the normalized area Lebesgue measure.

The aim of this paper is to characterize the boundedness and compactness of two Volterra type operators $I_{g}$ and $T_{g}$ from the analytic Morrey spaces $\mathcal{L}^{2, \lambda}$ to the classical Bloch space $B$, and from the little analytic Morrey spaces $\mathcal{L}_{0}^{2, \lambda}$ to the little Bloch space $B_{0}$. Also, we estimate the essential norm of $I_{g}$ and $T_{g}$.

The Morrey space was initially introduced in 1938 by Morrey [21] to show that certain systems of partial differential equations (PDEs) had Hölder continuous solutions. In the past, the Morrey space has been heavily studied in different areas. For example, Adams and Xiao studied Morrey spaces which is defined on Euclidean spaces $\mathbb{R}^{n}$ by potential theory and Hausdorff capacity in $[\mathbf{1}, \mathbf{2}]$. Cascante, Fàbrega, Ortega [11] (partially) and Wang and Xiao [28] studied holomorphic Campanato spaces on the open unit ball $\mathbb{B}^{n}$ of $\mathbb{C}^{n}$. But here we will be

2010 AMS Mathematics subject classification. Primary 47B38, 30H30, 30H99.

Keywords and phrases. Analytic Morrey space, Bloch space, Volterra type operator, essential norm.

The research was supported by the National Natural Science Foundation of China (grant No. 11271124) and the Natural Science Foundation of Fujian Province, China (grant No. 2015J01005).

The second author is the corresponding author.

Received by the editors on July 23, 2014. 
mostly interested in the analytic Morrey spaces $\mathcal{L}^{2, \lambda}$ in the unit disk. It was introduced and studied by $\mathrm{Wu}$ and Xie in [31].

For an $\operatorname{arc} I \subset \partial \mathbb{D}$, let $|I|=\frac{1}{2 \pi} \int_{I}|d \zeta|$ be the normalized arc length of $I$,

$$
f_{I}=\frac{1}{|I|} \int_{I} f(\zeta) \frac{|d \zeta|}{2 \pi}, \quad f \in H(\mathbb{D}),
$$

and let $S(I)$ be the Carleson box based on $I$ with

$$
S(I)=\left\{z \in \mathbb{D}: 1-|I| \leq|z|<1, \frac{z}{|z|} \in I\right\} .
$$

Clearly, if $I=\partial \mathbb{D}$, then $S(I)=\mathbb{D}$.

Let $\mathcal{L}^{2, \lambda}(\mathbb{D})$ represent the analytic Morrey spaces of all analytic functions $f \in H^{2}$ on $\mathbb{D}$ such that

$$
\sup _{I \subset \partial \mathbb{D}}\left(\frac{1}{|I|^{\lambda}} \int_{I}\left|f(\zeta)-f_{I}\right|^{2} \frac{|d \zeta|}{2 \pi}\right)^{1 / 2}<\infty,
$$

where $0<\lambda \leq 1$ and the Hardy space $H^{2}$ consist of analytic functions $f$ in $\mathbb{D}$ satisfying

$$
\sup _{0<r<1} \frac{1}{2 \pi} \int_{0}^{2 \pi}\left|f\left(r e^{i \theta}\right)\right|^{2} d \theta<\infty .
$$

Similarly to the relation between BMOA space and VMOA space, we have that $f \in \mathcal{L}_{0}^{2, \lambda}(\mathbb{D})$, the little Morrey spaces, if $f \in \mathcal{L}^{2, \lambda}(\mathbb{D})$ and

$$
\lim _{|I| \rightarrow 0}\left(\frac{1}{|I|^{\lambda}} \int_{I}\left|f(\zeta)-f_{I}\right|^{2} \frac{|d \zeta|}{2 \pi}\right)^{1 / 2}=0 .
$$

Xiao and $\mathrm{Xu}[36]$ studied the composition operators of $\mathcal{L}^{2, \lambda}$ spaces. Cascante, Fàbrega and Ortega [11] studied the Corona theorem of $\mathcal{L}^{2, \lambda}$. Wang and Xiao [29] characterized the first and second preduals of the analytic Morrey spaces. Xiao and Yuan [37] studied analytic Campanato spaces (including the analytic Morrey spaces) in terms of the Möbius mappings and the Littlewood-Paley forms. It is a useful tool for the study of harmonic analysis and partial differential equations. We refer the interested reader to $[\mathbf{2 1}, \mathbf{2 2}, \mathbf{4 2}]$.

The following lemma gives some equivalent conditions of $\mathcal{L}^{2, \lambda}(\mathbb{D})$ (see [32, Theorem 3.1] or [35, Theorem 3.21]). 
Lemma 1.1. Suppose that $0<\lambda<1$ and $f \in H(\mathbb{D})$. Let $a \in$ $\mathbb{D}, \varphi_{a}(z)=(a-z) /(1-\bar{a} z)$. Then the following statements are equivalent:

(i) $f \in \mathcal{L}^{2, \lambda}(\mathbb{D})$;

(ii) $\sup _{I \subset \partial \mathbb{D}} \frac{1}{|I|^{\lambda}} \int_{S(I)}\left|f^{\prime}(z)\right|^{2}\left(1-|z|^{2}\right) d m(z)<\infty$;

(iii) $\sup _{a \in \mathbb{D}}\left(1-|a|^{2}\right)^{1-\lambda} \int_{\mathbb{D}}\left|f^{\prime}(z)\right|^{2}\left(1-\left|\varphi_{a}(z)\right|^{2}\right) d m(z)<\infty$.

From the lemma above, we can define the norm of the function $f \in \mathcal{L}^{2, \lambda}(\mathbb{D})$ and its equivalent formula as follows:

$$
\begin{aligned}
\|f\|_{\mathcal{L}^{2, \lambda}} & =|f(0)|+\sup _{I \subset \partial \mathbb{D}}\left(\frac{1}{|I|^{\lambda}} \int_{S(I)}\left|f^{\prime}(z)\right|^{2}\left(1-|z|^{2}\right) d m(z)\right)^{1 / 2} \\
& \approx|f(0)|+\sup _{a \in \mathbb{D}}\left(\left(1-|a|^{2}\right)^{1-\lambda} \int_{\mathbb{D}}\left|f^{\prime}(z)\right|^{2}\left(1-\left|\varphi_{a}(z)\right|^{2}\right) d m(z)\right)^{1 / 2} .
\end{aligned}
$$

It is known that $\mathcal{L}^{2,1}(\mathbb{D})=\mathrm{BMOA}$ and if $0<\lambda<1$, BMOA $\subsetneq \mathcal{L}^{2, \lambda}(\mathbb{D})$. For more information on BMOA and VMOA, see [15].

A function $f$ analytic on the unit disk is said to belong to the Bloch space $B$ if

$$
\|f\|_{B}=\sup _{z \in \mathbb{D}}\left\{\left(1-|z|^{2}\right)\left|f^{\prime}(z)\right|\right\}<\infty,
$$

and to the little Bloch space $B_{0}$ if $f \in B$ and

$$
\lim _{|z| \rightarrow 1^{-}}\left(1-|z|^{2}\right)\left|f^{\prime}(z)\right|=0 .
$$

It is well known that $B$ is a Banach space under the norm $\|\mid f\|_{B}=$ $|f(0)|+\|f\|_{B}$ and $B_{0}$ is a closed subspace of $B$. See [5]. By [7, 34], together with [8, Lemma 2.1], we have the following equivalent statements about the norm of $f \in B$. 
Proposition 1.2. For all $p \in(1, \infty)$,

$$
\begin{aligned}
\left\||f \||_{B}\right. & \approx|f(0)|+\sup _{a \in \mathbb{D}}\left(\int_{\mathbb{D}}\left|f^{\prime}(z)\right|^{2}\left(1-\left|\varphi_{a}(z)\right|^{2}\right)^{p} d m(z)\right)^{1 / 2} \\
& \approx|f(0)|+\sup _{I \subset \partial \mathbb{D}}\left(\frac{1}{|I|^{p}} \int_{S(I)}\left|f^{\prime}(z)\right|^{2}\left(1-|z|^{2}\right)^{p} d m(z)\right)^{1 / 2} .
\end{aligned}
$$

Suppose that $g: \mathbb{D} \rightarrow \mathbb{C}$ is a holomorphic map. The integral operator $T_{g}$, called the Volterra-type operator, is defined as

$$
T_{g} f(z)=\int_{0}^{z} f(w) g^{\prime}(w) d w, \quad z \in \mathbb{D}, f \in H(\mathbb{D}) .
$$

In [23], Pommerenke introduced the operator $T_{g}$ and showed that $T_{g}$ is a bounded operator on the Hardy space $H^{2}$ if and only if $g \in$ BMOA.

The companion integral operator $I_{g}$ is analogously defined as

$$
I_{g} f(z)=\int_{0}^{z} f^{\prime}(w) g(w) d w, \quad z \in \mathbb{D}, f \in H(\mathbb{D}) .
$$

The boundedness, compactness or essential norm of $T_{g}$ and $I_{g}$ between spaces of analytic functions were investigated by many authors. Aleman and Siskakis in [4] studied the integral operator $T_{g}$ on the Bergman space, and then Aleman considered with Cima $T_{g}$ acting on the Hardy space in [3]. Siskakis and Zhao [27] also investigated $T_{g}$ on the BMOA space. $T_{g}$ on the $Q_{p}$ space was studied by Xiao in [33]. Li and Stević in [19] studied the boundedness and compactness of $T_{g}$ and $I_{g}$ on the Zygmund spaces and the little Zygmund spaces. Constantin in [12] considered the boundedness and compactness of $T_{g}$ on Fock spaces. Ye in [39] studied products of Volterra-type operators and composition operators on logarithmic Bloch space. Ye and Gao in [40] gave the boundedness and compactness of $T_{g}$ between different weighted Bloch spaces.

There are some articles about the integral operator acting on the Morrey space. For example, Wu in [30] considered $T_{g}$ from Hardy to analytic Morrey spaces. Li, Liu and Lou [17] characterized the boundedness and essential norms of $T_{g}$ and $I_{g}$ on analytic Morrey spaces (see also the related references therein). 
Now, we need two spaces. Let $\alpha>-1$. Recall that $f \in H(\mathbb{D})$ belongs to the weighted space $H_{\alpha}^{\infty}$ if it satisfies

$$
\sup _{z \in \mathbb{D}}\left(1-|z|^{2}\right)^{\alpha}|f(z)|<\infty .
$$

When $\alpha>-1, H_{\alpha}^{\infty}$ endowed with the norm $\|f\|_{\alpha}=\sup _{z \in \mathbb{D}}(1-$ $\left.|z|^{2}\right)^{\alpha}|f(z)|$ is a Banach space. This space is connected with the study of growth conditions of analytic functions and was also studied in detail, see $[\mathbf{9}, \mathbf{1 0}, \mathbf{2 5}, \mathbf{2 6}, \mathbf{3 8}]$. The space $H_{\alpha}^{\infty}$ is used in the characterizations of the boundedness and essential norm of $I_{g}$. Then we conclude the boundedness and essential norm of $T_{g}$ by introducing the following Bloch-Morrey type spaces.

Definition 1.3. Let $0<\lambda \leq 1$ and $p>1$. The Bloch-Morrey type space $B \mathcal{L}^{p, \lambda}$ is the set of all $g \in H(\mathbb{D})$ such that

$$
M(g)=\sup _{I \subset \partial \mathbb{D}}\left(\frac{1}{|I|^{p-\lambda+1}} \int_{S(I)}\left|g^{\prime}(z)\right|^{2}\left(1-|z|^{2}\right)^{p} d m(z)\right)^{1 / 2}<\infty .
$$

The corresponding subspace $B \mathcal{L}_{0}^{p, \lambda}$, the little Bloch-Morrey type space, can be defined as

$$
B \mathcal{L}_{0}^{p, \lambda}=\left\{g \in B \mathcal{L}^{p, \lambda}, \lim _{|I| \rightarrow 0}\left(\frac{1}{|I|^{p-\lambda+1}} \int_{S(I)}\left|g^{\prime}(z)\right|^{2}\left(1-|z|^{2}\right)^{p} d m(z)\right)^{1 / 2}=0\right\} .
$$

It is easy to prove that $B \mathcal{L}^{p, \lambda}$ is a Banach space under the norm

$$
\|g\|_{B \mathcal{L}^{p, \lambda}}=|g(0)|+M(g) .
$$

Clearly, $B \mathcal{L}^{p, 1}=B$. From [8], we know that $\|g\|_{B \mathcal{L}^{p, \lambda}}$ is comparable with the norm

$$
|g(0)|+\sup _{a \in \mathbb{D}}\left(\int_{\mathbb{D}}\left(\frac{1-|a|^{2}}{|1-\bar{a} z|^{2}}\right)^{p+1-\lambda}\left|g^{\prime}(z)\right|^{2}\left(1-|z|^{2}\right)^{p} d m(z)\right)^{1 / 2} .
$$

Notation. For two functions $F$ and $G$, if there is a constant $C>0$ dependent only on indexes $p, \lambda, \ldots$, such that $F \leq C G$, then we say that $F \lesssim G$. Furthermore, denote that $F \approx G$ ( $F$ is comparable with $G)$ whenever $F \lesssim G \lesssim F$. 
2. $\mathcal{L}^{2, \lambda}$ vs $B$. Evidently, when $0<\lambda<1$, BMOA $\subsetneq \mathcal{L}^{2, \lambda}(\mathbb{D})$. On the other hand BMOA $\subsetneq B$. Does $\mathcal{L}^{2, \lambda}$ and $B$ have the inclusion relation? We claim that the answer is negative by the following two proposition.

Proposition 2.1. $\mathcal{L}^{2, \lambda} \nsubseteq B$.

Proof. Consider $g(z)=\left(\log \frac{1}{1-z}\right)^{2}$, which is obviously not a Bloch function. We get that $g(z) \in \mathcal{L}^{2, \lambda}$. Indeed,

$$
\begin{aligned}
\sup _{a \in \mathbb{D}}\left(1-|a|^{2}\right)^{1-\lambda} & \int_{\mathbb{D}}\left|g^{\prime}(z)\right|^{2}\left(1-\left|\varphi_{a}(z)\right|^{2}\right) d m(z) \\
& \lesssim \sup _{a \in \mathbb{D}}\left(1-|a|^{2}\right)^{1-\lambda} \\
& \times \int_{\mathbb{D}}\left|\frac{1}{1-z}\right|^{2} \log ^{2} \frac{1}{1-|z|}\left(1-\left|\varphi_{a}(z)\right|^{2}\right) d m(z) \\
& \lesssim \int_{\mathbb{D}}\left|\frac{1}{1-z}\right|^{2}\left(1-|z|^{2}\right)^{1-\lambda} \log ^{2} \frac{1}{1-|z|} d m(z) \\
& =\int_{0}^{1} \int_{0}^{2 \pi}\left|\frac{1}{1-r e^{i \theta}}\right|^{2} d \theta\left(1-r^{2}\right)^{1-\lambda} \log ^{2} \frac{1}{1-r} d r \\
& =\int_{0}^{1}\left(1-r^{2}\right)^{-\lambda} \log ^{2} \frac{1}{1-r} d r<\infty .
\end{aligned}
$$

This finishes the proof.

Conversely, the function

$$
f(z)=\sum_{n=0}^{\infty} z^{2^{n}}
$$

is a Bloch function (see [6]), and it is well known that it has a radial limit almost nowhere. Consequently, $f$ does not belong to any of the Hardy spaces, and so $f \notin \mathcal{L}^{2, \lambda}$. Now we have the following proposition.

Proposition 2.2. $B \nsubseteq \mathcal{L}^{2, \lambda}$. 
3. Boundedness of $I_{g}$ and $T_{g}$ from $\mathcal{L}^{2, \lambda}$ to $B$. In this section, we prove the boundedness and estimate the norms of $I_{g}$ and $T_{g}$. The following lemmas will be used through this paper.

Lemma 3.1. Let $0<\lambda<1$ and $b \in \mathbb{D}$. We set functions $f_{b}(z)$ and $F_{b}(z)$ as

$f_{b}(z)=\left(1-|b|^{2}\right)^{(1-\lambda) / 2}\left(\varphi_{b}(z)-b\right), F_{b}(z)=\left(1-|b|^{2}\right)(1-\bar{b} z)^{(\lambda-3) / 2}$.

Then $f_{b}(z) \in \mathcal{L}^{2, \lambda}(\mathbb{D})$ and $F_{b}(z) \in \mathcal{L}^{2, \lambda}(\mathbb{D})$. Particularly, we have $f_{b}(z) \in \mathcal{L}_{0}^{2, \lambda}(\mathbb{D})$ and $F_{b}(z) \in \mathcal{L}_{0}^{2, \lambda}(\mathbb{D})$. Moreover, $\left\|f_{b}\right\|_{\mathcal{L}^{2, \lambda}} \lesssim 1$, $\left\|F_{b}\right\|_{\mathcal{L}^{2, \lambda}} \lesssim 1$.

Proof. See [17, Lemma 4]. From its proof, we further deduce that $f_{b}(z) \in \mathcal{L}_{0}^{2, \lambda}(\mathbb{D})$ and $F_{b}(z) \in \mathcal{L}_{0}^{2, \lambda}(\mathbb{D})$.

We get a result about the growth rate of functions in $\mathcal{L}^{2, \lambda}(\mathbb{D})$ from $[17]$.

Lemma 3.2. Let $0<\lambda<1$. If $f \in \mathcal{L}^{2, \lambda}(\mathbb{D})$, then

$$
|f(z)| \lesssim \frac{\|f\|_{\mathcal{L}^{2, \lambda}}}{\left(1-|z|^{2}\right)^{(1-\lambda) / 2}}, \quad z \in \mathbb{D}
$$

We first consider the boundedness of $I_{g}: \mathcal{L}^{2, \lambda} \rightarrow B$.

Theorem 3.3. Let $0<\lambda<1$ and $g \in H(\mathbb{D})$. Then $I_{g}: \mathcal{L}^{2, \lambda} \rightarrow B$ is bounded if and only if $g \in H_{(\lambda-1) / 2}^{\infty}$. Moreover, the operator norm satisfies

$$
\left\|I_{g}\right\| \approx\|g\|_{(\lambda-1) / 2}
$$

Proof. For $0<\lambda<1$ and $1<2-\lambda$, we set $B=Q_{2-\lambda}$. 
Let $g \in H_{(\lambda-1) / 2}^{\infty}$. For any $f \in \mathcal{L}^{2, \lambda}(\mathbb{D})$, we have

$$
\begin{aligned}
\left\|I_{g} f\right\|_{B} & \approx \sup _{a \in \mathbb{D}}\left(\int_{\mathbb{D}}\left|f^{\prime}(z)\right|^{2}|g(z)|^{2}\left(1-\left|\varphi_{a}(z)\right|^{2}\right)^{2-\lambda} d m(z)\right)^{1 / 2} \\
& =\sup _{a \in \mathbb{D}}\left(\left(1-|a|^{2}\right)^{1-\lambda} \int_{\mathbb{D}}\left|f^{\prime}(z)\right|^{2}\right. \\
& \left.\times\left(1-\left|\varphi_{a}(z)\right|^{2}\right)|g(z)|^{2}\left(\frac{1-|z|^{2}}{|1-\bar{a} z|^{2}}\right)^{1-\lambda} d m(z)\right)^{1 / 2} \\
& \lesssim\|g\|_{(\lambda-1) / 2}\|f\|_{\mathcal{L}^{2, \lambda}},
\end{aligned}
$$

which implies that $I_{g}$ is bounded and $\left\|I_{g}\right\| \lesssim\|g\|_{(\lambda-1) / 2}$.

On the other hand, let $I_{g}$ be bounded. For any $b \in \mathbb{D}$, considering functions $f_{b}(z)$ in Lemma 3.1 , we have $\left\|f_{b}\right\|_{\mathcal{L}^{2, \lambda}} \lesssim 1$. Then

$$
\begin{aligned}
\left\|I_{g}\right\| & \gtrsim\left\|I_{g} f_{b}\right\|_{B} \\
& \approx \sup _{a \in \mathbb{D}}\left(\int_{\mathbb{D}}\left|f_{b}^{\prime}(z)\right|^{2}|g(z)|^{2}\left(1-\left|\varphi_{a}(z)\right|^{2}\right)^{2-\lambda} d m(z)\right)^{1 / 2} \\
& =\sup _{a \in \mathbb{D}}\left(\int_{\mathbb{D}} \frac{\left(1-|b|^{2}\right)^{1+\lambda}}{|1-\bar{b} z|^{4}}|g(z)|^{2}\left(1-\left|\varphi_{a}(z)\right|^{2}\right)^{1+1-\lambda} d m(z)\right)^{1 / 2} \\
(3.1) & \geqslant\left(\int_{\mathbb{D}} \frac{\left(1-|b|^{2}\right)^{2}}{|1-\bar{b} z|^{4}}|g(z)|^{2}\left(\frac{1-|z|^{2}}{|1-\bar{b} z|^{2}}\right)^{1-\lambda}\left(1-\left|\varphi_{b}(z)\right|^{2}\right) d m(z)\right)^{1 / 2} \\
& =\left(\int_{\mathbb{D}}\left|\varphi_{b}^{\prime}(z)\right|^{2}|g(z)|^{2}\left(\frac{1-|z|^{2}}{|1-\bar{b} z|^{2}}\right)^{1-\lambda}\left(1-\left|\varphi_{b}(z)\right|^{2}\right) d m(z)\right)^{1 / 2} \\
& =\left(\int_{\mathbb{D}}\left|g\left(\varphi_{b}(w)\right)\right|^{2}\left(\frac{1-\left|\varphi_{b}(w)\right|^{2}}{\left|1-\bar{b} \varphi_{b}(w)\right|^{2}}\right)^{1-\lambda}\left(1-|w|^{2}\right) d m(w)\right)^{1 / 2} \\
& \gtrsim\left|\frac{g(b)}{\left(1-|b|^{2}\right)^{(1-\lambda) / 2} \mid,}\right|
\end{aligned}
$$

where we used [41, Lemma 4.12] in the last inequality. Since $b$ is arbitrary, we have $\left\|I_{g}\right\| \gtrsim\|g\|_{(\lambda-1) / 2}$. The proof is finished.

With the space $B \mathcal{L}^{p, \lambda}$, we can establish the boundedness of $T_{g}$ : $\mathcal{L}^{2, \lambda} \rightarrow B$ as in the following theorem. 
Theorem 3.4. Suppose that $0<\lambda<1$ and $g \in H(\mathbb{D})$. Then the following conditions are equivalent:

(i) $T_{g}: \mathcal{L}^{2, \lambda} \rightarrow B$ is bounded;

(ii) $g \in B \mathcal{L}^{p, \lambda}$ for all $p \in(1, \infty)$;

(iii) $g \in B \mathcal{L}^{p, \lambda}$ for some $p \in(1, \infty)$.

Moreover,

$$
\left\|T_{g}\right\| \approx M(g)
$$

Proof. (i) $\Rightarrow$ (ii). Suppose that $T_{g}: \mathcal{L}^{2, \lambda} \rightarrow B$ is bounded. For any $I \subset \partial \mathbb{D}$, let $b=(1-|I|) \zeta \in \mathbb{D}$, where $\zeta$ is the center of $I$. Then

$$
\left(1-|b|^{2}\right) \approx|1-\bar{b} z| \approx|I|, \quad z \in S(I)
$$

Considering the functions $F_{b}(z)$ in Lemma 3.1 , we have $\left\|F_{b}\right\|_{\mathcal{L}^{2, \lambda}} \lesssim 1$. This, together with Proposition 2.2, we obtain that for any $p \in(1, \infty)$,

$$
\begin{aligned}
\frac{1}{|I|^{p-\lambda+1}} & \int_{S(I)}\left|g^{\prime}(z)\right|^{2}\left(1-|z|^{2}\right)^{p} d m(z) \\
& \approx \frac{1}{|I|^{p}} \int_{S(I)}\left|F_{b}(z)\right|^{2}\left|g^{\prime}(z)\right|^{2}\left(1-|z|^{2}\right)^{p} d m(z) \\
& \lesssim\left\|T_{g} F_{b}\right\|_{B}^{2} \leq\left\|T_{g}\right\|^{2}\left\|F_{b}\right\|_{\mathcal{L}^{2, \lambda}}^{2} \lesssim\left\|T_{g}\right\|^{2}
\end{aligned}
$$

Since $I$ is arbitrary, we have $M(g) \lesssim\left\|T_{g}\right\|$.

(ii) $\Rightarrow$ (iii). It is obvious.

(iii) $\Rightarrow$ (i). Suppose that fixed $p \in(1, \infty)$ and $M(g)<\infty$. For $f \in \mathcal{L}^{2, \lambda}(\mathbb{D})$ and any $I \subset \partial \mathbb{D}$, by Lemma 3.2, it follows that

$$
\begin{aligned}
\left\|T_{g} f\right\|_{B} & \approx \sup _{I \subset \partial \mathbb{D}}\left(\frac{1}{|I|^{p}} \int_{S(I)}|f(z)|^{2}\left|g^{\prime}(z)\right|^{2}\left(1-|z|^{2}\right)^{p} d m(z)\right)^{1 / 2} \\
& \lesssim\|f\|_{\mathcal{L}^{2, \lambda}} \cdot \sup _{I \subset \partial \mathbb{D}}\left(\frac{1}{|I|^{p}} \int_{S(I)}\left|g^{\prime}(z)\right|^{2}\left(1-|z|^{2}\right)^{p+\lambda-1} d m(z)\right)^{1 / 2}
\end{aligned}
$$

To the end, for a given subarc $I$ of $\partial \mathbb{D}$, let $\mathcal{D}_{n}(I)$ represent the set of $2^{n}$ subarcs of length $2^{-n}|I|$ obtained by $n$ successive bipartition of $I$. For each $J \in \mathcal{D}_{n}(I)$, write $T(J)$ for the top half Carleson box of $S(J)$, 
i.e.,

$$
T(J)=\left\{z \in S(J): \frac{z}{|z|} \in J, 1-|J|<|z|<1-\frac{|J|}{2}\right\} .
$$

Then

$$
S(I)=\bigcup_{n=0}^{\infty} \bigcup_{J \in \mathcal{D}_{n}(I)} T(J) .
$$

Noting that $z \in T(J), 1-|z| \approx|J|$, one has

$$
\begin{aligned}
& \int_{S(I)}\left|g^{\prime}(z)\right|^{2}\left(1-|z|^{2}\right)^{p+\lambda-1} d m(z) \\
& =\sum_{n=0}^{\infty} \sum_{J \in \mathcal{D}_{n}(I)} \int_{T(J)}\left|g^{\prime}(z)\right|^{2}\left(1-|z|^{2}\right)^{p+\lambda-1} d m(z) \\
& \approx \sum_{n=0}^{\infty} \sum_{J \in \mathcal{D}_{n}(I)} \int_{T(J)}|J|^{\lambda-1}\left|g^{\prime}(z)\right|^{2}\left(1-|z|^{2}\right)^{p} d m(z) \\
& \leq \sum_{n=0}^{\infty} \sum_{J \in \mathcal{D}_{n}(I)} \int_{S(J)}|J|^{\lambda-1}\left|g^{\prime}(z)\right|^{2}\left(1-|z|^{2}\right)^{p} d m(z) \\
& \leq \sum_{n=0}^{\infty} \sum_{J \in \mathcal{D}_{n}(I)} M(g)^{2}|J|^{\lambda-1}|J|^{p-\lambda+1} \\
& =\sum_{n=0}^{\infty} 2^{n} M(g)^{2}|J|^{p}=\sum_{n=0}^{\infty}\left(2^{n}\right)^{1-p} M(g)^{2}|I|^{p} \\
& \lesssim M(g)^{2}|I|^{p} .
\end{aligned}
$$

Now invoking (3.2),

$$
\left\|T_{g} f\right\|_{B} \lesssim M(g) \cdot\|f\|_{\mathcal{L}^{2, \lambda} .}
$$

As a result, $\left\|T_{g}\right\| \lesssim M(g)$.

Theorem 3.4 has an interesting consequence.

Corollary 3.5. Let $0<\lambda<1$ and $1<p<q<\infty$. Then $B \mathcal{L}^{p, \lambda}=B \mathcal{L}^{q, \lambda}$. 
4. Essential norm of $I_{g}$ and $T_{g}$ from $\mathcal{L}^{2, \lambda}$ to $B$. Let $X$ and $Y$ be Banach spaces. The essential norm of a bounded operator $T: X \rightarrow Y$, $\|T\|_{e, X \rightarrow Y}$, is defined as the distance from $T$ to the space of compact operators,

$$
\|T\|_{e, X \rightarrow Y}=\inf \left\{\|T-K\|_{X \rightarrow Y}: K \text { is any compact operator }\right\},
$$

where the norm of $T$ is denoted by $\|\cdot\|_{X \rightarrow Y}$.

Since that $T$ is compact if and only if $\|T\|_{e, X \rightarrow Y}=0$, then the estimation of $\|T\|_{e, X \rightarrow Y}$ indicates the condition for $T$ to be compact. For some recent results related to the essential norm, see $[\mathbf{1 6}, \mathbf{1 7}, \mathbf{1 8}$, 24] and the references therein.

In this section, we estimate the essential norm of $I_{g}$ and $T_{g}$ from $\mathcal{L}^{2, \lambda}$ to $B$. We need some auxiliary results.

Lemma 4.1. Let $0<\lambda<1$. For $0<t<1, z \in \mathbb{D}, f_{t}(z)=f(t z)$. If $f \in \mathcal{L}^{2, \lambda}(\mathbb{D})$, then $f_{t} \in \mathcal{L}_{0}^{2, \lambda}(\mathbb{D})$ and $\left\|f_{t}\right\|_{\mathcal{L}^{2, \lambda}} \leq\|f\|_{\mathcal{L}^{2, \lambda}}$.

Proof. If $f \in \mathcal{L}^{2, \lambda}(\mathbb{D})$ and $0<t<1$, then $f_{t}$ is analytic on the closed unit disk $\overline{\mathbb{D}}$. A simple computation shows that $f_{t} \in \mathcal{L}_{0}^{2, \lambda}(\mathbb{D})$. In addition, by the Poisson formula, we have

$$
f_{t}(z)=\int_{0}^{2 \pi} f\left(z e^{i \theta}\right) \frac{1-t^{2}}{\left|e^{i \theta}-t\right|^{2}} \frac{d \theta}{2 \pi}, \quad z \in \mathbb{D} .
$$

Then,

$$
\begin{aligned}
\sup _{a \in \mathbb{D}}(1 & \left.-|a|^{2}\right)^{1-\lambda} \int_{\mathbb{D}}\left|f_{t}^{\prime}(z)\right|^{2}\left(1-\left|\varphi_{a}(z)\right|^{2}\right) d m(z) \\
\leq & \sup _{a \in \mathbb{D}}\left(1-|a|^{2}\right)^{1-\lambda} \\
& \times \int_{\mathbb{D}} \int_{0}^{2 \pi}\left|f^{\prime}\left(z e^{i \theta}\right)\right|^{2} \frac{1-t^{2}}{\left|e^{i \theta}-t\right|^{2}} \frac{d \theta}{2 \pi}\left(1-\left|\varphi_{a}(z)\right|^{2}\right) d m(z) \\
= & \int_{0}^{2 \pi} \sup _{a \in \mathbb{D}}\left(1-|a|^{2}\right)^{1-\lambda} \int_{\mathbb{D}}\left|f^{\prime}\left(z e^{i \theta}\right)\right|^{2} \\
& \left(1-\left|\varphi_{a}(z)\right|^{2}\right) d m(z) \frac{1-t^{2}}{\left|e^{i \theta}-t\right|^{2}} \frac{d \theta}{2 \pi} \\
\leq & \sup _{a \in \mathbb{D}}\left(1-|a|^{2}\right)^{1-\lambda} \int_{\mathbb{D}}\left|f^{\prime}(z)\right|^{2}\left(1-\left|\varphi_{a}(z)\right|^{2}\right) d m(z)
\end{aligned}
$$




$$
\begin{aligned}
& \int_{0}^{2 \pi} \frac{1-t^{2}}{\left|e^{i \theta}-t\right|^{2}} \frac{d \theta}{2 \pi} \\
& =\sup _{a \in \mathbb{D}}\left(1-|a|^{2}\right)^{1-\lambda} \int_{\mathbb{D}}\left|f^{\prime}(z)\right|^{2}\left(1-\left|\varphi_{a}(z)\right|^{2}\right) d m(z) .
\end{aligned}
$$

Thus, $\left\|f_{t}\right\|_{\mathcal{L}^{2, \lambda}} \leq\|f\|_{\mathcal{L}^{2, \lambda}}$.

By Lemma 3.2 and standard arguments (see, e.g., [13, Proposition 3.11]), the following lemma follows.

Lemma 4.2. Assume that $g$ is an analytic function on $\mathbb{D}$. Then $T_{g}$ $\left(\right.$ or $\left.I_{g}\right): \mathcal{L}^{2, \lambda} \rightarrow B$ is compact if and only if $T_{g}\left(\right.$ or $\left.I_{g}\right): \mathcal{L}^{2, \lambda} \rightarrow B$ is bounded, and for any bounded sequence $\left(f_{k}\right)_{k \in \mathbb{N}}$ in $\mathcal{L}^{2, \lambda}$ which converges to zero uniformly on $\mathbb{D}$ as $k \rightarrow \infty,\left\|T_{g} f_{k}\right\|_{B} \rightarrow 0\left(\right.$ or $\left.\left\|I_{g} f_{k}\right\|_{B} \rightarrow 0\right)$ as $k \rightarrow \infty$.

Lemma 4.3. Suppose that $0<\lambda<1$ and $p>1$. For $g \in B \mathcal{L}^{p, \lambda}$, define the following operators $T_{g, r}: \mathcal{L}^{2, \lambda} \rightarrow B$ :

$$
T_{g, r} f(z)=\int_{0}^{z} f(r w) g^{\prime}(w) d w
$$

where $r \in(0,1)$. Then $T_{g, r}$ is compact.

Proof. Let $\left\{f_{n}\right\}$ be such that $\left\|f_{n}\right\|_{\mathcal{L}^{2, \lambda}} \leq 1$ and $f_{n} \rightarrow 0$ uniformly on compact subsets of $\mathbb{D}$ as $n \rightarrow \infty$. We are required to show that $\lim _{n \rightarrow \infty}\left\|T_{g, r} f_{n}\right\|_{B}=0$. In fact, since $\|g\|_{B} \lesssim M(g)$, we have

$$
\left|g^{\prime}(z)\right| \lesssim \frac{M(g)}{1-|z|^{2}} .
$$

From $\left\|f_{n}\right\|_{\mathcal{L}^{2, \lambda}} \leq 1$ and Lemma 3.2 , it yields that $\left(1-|r|^{2}\right)^{(1-\lambda) / 2}\left|f_{n}(r z)\right|$ $\lesssim 1$. Thus,

$$
\begin{aligned}
\left\|T_{g, r} f_{n}\right\|_{B} & =\sup _{z \in \mathbb{D}}\left(1-|z|^{2}\right)\left|f_{n}(r z)\right|\left|g^{\prime}(z)\right| \\
& \lesssim M(g) \sup _{z \in \mathbb{D}} \frac{1}{\left(1-r^{2}\right)^{(1-\lambda) / 2}} .
\end{aligned}
$$

Accordingly, by the dominated convergence theorem, one reaches

$$
\lim _{n \rightarrow \infty}\left\|T_{g_{r}} f_{n}\right\|_{B}=0 .
$$


Now, we present the main result of this section.

Theorem 4.4. Suppose $0<\lambda<1$ and $g \in H(\mathbb{D})$. If $I_{g}: \mathcal{L}^{2, \lambda} \rightarrow B$ is bounded, then

$$
\left\|I_{g}\right\|_{e, \mathcal{L}^{2, \lambda} \rightarrow B} \approx\|g\|_{(\lambda-1) / 2} .
$$

Proof. Choose the zero operator $O: \mathcal{L}^{2, \lambda} \rightarrow B: f \mapsto 0$. Since $O$ is compact and $\|O\|=0$, we get

$$
\left\|I_{g}\right\|_{e, \mathcal{L}^{2, \lambda} \rightarrow B}=\inf _{K}\left\|I_{g}-K\right\| \leq\left\|I_{g}\right\| \lesssim\|g\|_{(\lambda-1) / 2} .
$$

Conversely, choose the sequence $\left\{b_{n}\right\} \subset \mathbb{D}$ such that $\left|b_{n}\right| \rightarrow 1$ as $n \rightarrow \infty$. Considering the sequence of functions $f_{n}(z)=(1-$ $\left.\left|b_{n}\right|^{2}\right)^{(1-\lambda) / 2}\left(\varphi_{b_{n}}(z)-b_{n}\right)$, we obtain $\left\|f_{n}\right\|_{\mathcal{L}^{2, \lambda}} \lesssim 1$ by Lemma 3.1. By an easy calculation,

$$
f_{n}(z)=-\left(1-\left|b_{n}\right|^{2}\right)^{(1+\lambda) / 2} \int_{0}^{z} \frac{d w}{\left(1-\overline{b_{n}} z\right)^{2}},
$$

and thus $f_{n}$ converges to zero uniformly on compact subsets of $\mathbb{D}$. Then $\left\|K f_{n}\right\|_{B} \rightarrow 0$ as $n \rightarrow \infty$ for any compact operator $K$. So

$$
\begin{aligned}
\left\|I_{g}-K\right\| & \gtrsim \limsup _{n \rightarrow \infty}\left\|\left(I_{g}-K\right) f_{n}\right\|_{B} \\
& \geq \limsup _{n \rightarrow \infty}\left(\left\|I_{g} f_{n}\right\|_{B}-\left\|K f_{n}\right\|_{B}\right) \\
& \geq \limsup _{n \rightarrow \infty}\left\|I_{g} f_{n}\right\|_{B} .
\end{aligned}
$$

By (3.1), we have

$$
\left\|I_{g}-K\right\| \gtrsim \limsup _{n \rightarrow \infty}\left|\frac{g\left(b_{n}\right)}{\left(1-\left|b_{n}\right|^{2}\right)^{(1-\lambda) / 2}}\right| .
$$

Then the arbitrary choice of the sequence $\left\{b_{n}\right\}$ implies

$$
\left\|I_{g}\right\|_{e, \mathcal{L}^{2, \lambda} \rightarrow B} \gtrsim\|g\|_{(\lambda-1) / 2} \text {. }
$$

Theorem 4.5. Suppose $0<\lambda<1$ and $g \in H(\mathbb{D})$. If $T_{g}: \mathcal{L}^{2, \lambda} \rightarrow B$ is bounded, then

$$
\left\|T_{g}\right\|_{e, \mathcal{L}^{2, \lambda} \rightarrow B} \approx \limsup _{|a| \rightarrow 1}\left(\int_{\mathbb{D}}\left(\frac{1-|a|^{2}}{|1-\bar{a} z|^{2}}\right)^{p+1-\lambda}\left|g^{\prime}(z)\right|^{2}\left(1-|z|^{2}\right)^{p} d m(z)\right)^{1 / 2} .
$$


Proof. For any $r_{n} \in(0,1)$ such that $r_{n} \rightarrow 1$ as $n \rightarrow \infty$, we introduce $T_{g, r_{n}}: \mathcal{L}^{2, \lambda} \rightarrow B$ which is compact. Letting $s \in(0,1)$, we have

$$
\begin{aligned}
& \left\|T_{g}\right\|_{e, \mathcal{L}^{2, \lambda} \rightarrow B} \leq\left\|T_{g}-T_{g, r_{n}}\right\| \approx \sup _{\|f\|_{\mathcal{L}^{2, \lambda}}=1}\left\|T_{g}-T_{g, r_{n}}\right\|_{B} \\
& =\sup _{\|f\|_{\mathcal{L}^{2, \lambda}}=1} \\
& \times \sup _{a \in \mathbb{D}}\left(\int_{\mathbb{D}}\left|f(z)-f\left(r_{n} z\right)\right|^{2}\left|g^{\prime}(z)\right|^{2}\left(1-\left|\varphi_{a}(z)\right|^{2}\right)^{p+1-\lambda} d m(z)\right)^{1 / 2} \\
& \leq \sup _{\|f\|_{\mathcal{L}^{2, \lambda}}=1} \\
& \times \sup _{|a| \leq s}\left(\int_{\mathbb{D}}\left|f(z)-f\left(r_{n} z\right)\right|^{2}\left|g^{\prime}(z)\right|^{2}\left(1-\left|\varphi_{a}(z)\right|^{2}\right)^{p+1-\lambda} d m(z)\right)^{1 / 2} \\
& \quad+\sup _{\|f\|_{\mathcal{L}^{2, \lambda}}=1} \\
& \times \sup _{|a|>s}\left(\int_{\mathbb{D}}\left|f(z)-f\left(r_{n} z\right)\right|^{2}\left|g^{\prime}(z)\right|^{2}\left(1-\left|\varphi_{a}(z)\right|^{2}\right)^{p+1-\lambda} d m(z)\right)^{1 / 2} \\
& \leq \sup _{\|f\|_{\mathcal{L}^{2, \lambda}}=1} \\
& \quad \sup _{|a| \leq s}\left(\int_{\mathbb{D}}\left|f(z)-f\left(r_{n} z\right)\right|^{2}\left|g^{\prime}(z)\right|^{2}\left(1-\left|\varphi_{a}(z)\right|^{2}\right)^{p+1-\lambda} d m(z)\right)^{1 / 2} \\
& \quad+2 \sup _{|a|>s}\left(\int_{\mathbb{D}}\left(\frac{1-|a|^{2}}{|1-\bar{a} z|^{2}}\right)^{p+1-\lambda}\left|g^{\prime}(z)\right|^{2}\left(1-|z|^{2}\right)^{p} d m(z)\right)^{1 / 2} \\
& \triangleq K_{1}+K_{2} \cdot
\end{aligned}
$$

Since $|a| \leq s$ is a closed set of $\mathbb{D}$ and $g \in B \mathcal{L}^{p, \lambda}$, the dominated convergence theorem yields $K_{1} \rightarrow 0$ as $n \rightarrow \infty$.

Now, letting $n \rightarrow \infty$ and then letting $s \rightarrow 1$, we get

$$
\begin{aligned}
& \left\|T_{g}\right\|_{e, \mathcal{L}^{2, \lambda} \rightarrow B} \\
& \quad \lesssim \limsup _{|a| \rightarrow 1}\left(\int_{\mathbb{D}}\left(\frac{1-|a|^{2}}{|1-\bar{a} z|^{2}}\right)^{p+1-\lambda}\left|g^{\prime}(z)\right|^{2}\left(1-|z|^{2}\right)^{p} d m(z)\right)^{1 / 2} .
\end{aligned}
$$

Conversely, let $I_{n} \subset \partial \mathbb{D}$ be such that $\left|I_{n}\right| \rightarrow 0$ as $n \rightarrow \infty$. $\zeta_{n}$ is the 
center of arc $I$ and $b_{n}=\left(1-\left|I_{n}\right|\right) \zeta$, so

$$
\left(1-\left|b_{n}\right|^{2}\right) \approx\left|1-\overline{b_{n}} z\right| \approx\left|I_{n}\right|, \quad z \in S\left(I_{n}\right) .
$$

Consider the function $F_{n}(z)=\left(1-\left|b_{n}\right|^{2}\right)\left(1-\overline{b_{n}} z\right)^{(\lambda-3) / 2}$. Then $\left\|F_{n}\right\|_{\mathcal{L}^{2, \lambda}} \lesssim 1$ and $F_{n} \rightarrow 0$ uniformly on the compact subsets of $\mathbb{D}$ as $n \rightarrow \infty$ by Lemma 3.1. Thus, $\left\|K F_{n}\right\|_{B} \rightarrow 0$ for any compact operator $K$. Therefore,

$$
\begin{aligned}
\left\|T_{g}-K\right\| & \gtrsim \limsup _{n \rightarrow \infty}\left\|\left(T_{g}-K\right) F_{n}\right\|_{B} \\
& \geq \limsup _{n \rightarrow \infty}\left(\left\|T_{g} F_{n}\right\|_{B}-\left\|K F_{n}\right\|_{B}\right) \\
& \geq \limsup _{n \rightarrow \infty}\left\|T_{g} f_{n}\right\|_{B} \\
& \geq \limsup _{n \rightarrow \infty}\left(\frac{1}{\left|I_{n}\right|^{p}} \int_{S\left(I_{n}\right)}\left|F_{n}(z)\right|^{2}\left|g^{\prime}(z)\right|^{2}\left(1-|z|^{2}\right)^{p} d m(z)\right)^{1 / 2} \\
& \approx \limsup _{n \rightarrow \infty}\left(\frac{1}{\left|I_{n}\right|^{p+1-\lambda}} \int_{S\left(I_{n}\right)}\left|g^{\prime}(z)\right|^{2}\left(1-|z|^{2}\right)^{p} d m(z)\right)^{1 / 2}
\end{aligned}
$$

Since the sequence $\left\{I_{n}\right\}$ is arbitrary, we conclude

$$
\begin{aligned}
& \left\|T_{g}\right\|_{e, \mathcal{L}^{2, \lambda} \rightarrow B} \\
& \gtrsim \limsup _{|I| \rightarrow 0}\left(\frac{1}{|I|^{p+1-\lambda}} \int_{S(I)}\left|g^{\prime}(z)\right|^{2}\left(1-|z|^{2}\right)^{p} d m(z)\right)^{1 / 2} \\
& \approx \limsup _{|a| \rightarrow 1}\left(\int_{\mathbb{D}}\left(\frac{1-|a|^{2}}{|1-\bar{a} z|^{2}}\right)^{p+1-\lambda}\left|g^{\prime}(z)\right|^{2}\left(1-|z|^{2}\right)^{p} d m(z)\right)^{1 / 2}
\end{aligned}
$$

This completes the proof.

We have the following corollary about their compactness.

Corollary 4.6. Suppose that $0<\lambda<1$ and $p>1$. Then:

(i) $I_{g}: \mathcal{L}^{2, \lambda} \rightarrow B$ is compact if and only if $g=0$.

(ii) $T_{g}: \mathcal{L}^{2, \lambda} \rightarrow B$ is compact if and only if $g \in B \mathcal{L}_{0}^{p, \lambda}$. 
5. Boundedness and essential norm of $I_{g}$ and $T_{g}$ from $\mathcal{L}_{0}^{2, \lambda}$ to $B_{0}$.

Theorem 5.1. Let $0<\lambda<1$ and $g \in H(\mathbb{D})$. Then $I_{g}: \mathcal{L}_{0}^{2, \lambda} \rightarrow B_{0}$ is bounded if and only if $g \in H_{(\lambda-1) / 2}^{\infty}$. Moreover,

$$
\left\|I_{g}\right\| \approx\|g\|_{(\lambda-1) / 2} .
$$

Proof. Necessity. Assume that $I_{g}: \mathcal{L}_{0}^{2, \lambda} \rightarrow B_{0}$ is bounded. Then it is clear that $I_{g}: \mathcal{L}_{0}^{2, \lambda} \rightarrow B$ is bounded. The necessity of Theorem 3.3, together with $f_{b}(z) \in \mathcal{L}_{0}^{2, \lambda}(\mathbb{D})$, proves $g \in H_{(\lambda-1) / 2}^{\infty}$.

Sufficiency. Let $g \in H_{(\lambda-1) / 2}^{\infty}$. Then, from Theorem $3.3, I_{g}: \mathcal{L}^{2, \lambda} \rightarrow$ $B$ is bounded, and hence $I_{g}: \mathcal{L}_{0}^{2, \lambda} \rightarrow B$ is bounded. It suffices to prove that, for any $f \in \mathcal{L}_{0}^{2, \lambda}, I_{g} f \in B_{0}$. In fact, for any $f \in \mathcal{L}_{0}^{2, \lambda}$, we have

$$
\begin{aligned}
& \lim _{|a| \rightarrow 1} \int_{\mathbb{D}}\left|f^{\prime}(z)\right|^{2}|g(z)|^{2}\left(1-\left|\varphi_{a}(z)\right|^{2}\right)^{2-\lambda} d m(z) \\
& =\lim _{|a| \rightarrow 1}\left(1-|a|^{2}\right)^{1-\lambda} \\
& \quad \times \int_{\mathbb{D}}\left|f^{\prime}(z)\right|^{2}\left(1-\left|\varphi_{a}(z)\right|^{2}\right)|g(z)|^{2}\left(\frac{1-|z|^{2}}{|1-\bar{a} z|^{2}}\right)^{1-\lambda} d m(z) \\
& \leq\|g\|_{(\lambda-1) / 2} \cdot \lim _{|a| \rightarrow 1}\left(1-|a|^{2}\right)^{1-\lambda} \\
& \quad \times \int_{\mathbb{D}}\left|f^{\prime}(z)\right|^{2}\left(1-\left|\varphi_{a}(z)\right|^{2}\right) d m(z)=0 .
\end{aligned}
$$

Consequently, $I_{g}: \mathcal{L}_{0}^{2, \lambda} \rightarrow B_{0}$ is bounded.

Theorem 5.2. Suppose that $0<\lambda<1$ and $g \in H(\mathbb{D})$. If $I_{g}: \mathcal{L}_{0}^{2, \lambda} \rightarrow$ $B_{0}$ is bounded, then

$$
\left\|I_{g}\right\|_{e, \mathcal{L}_{0}^{2, \lambda} \rightarrow B_{0}} \approx\|g\|_{(\lambda-1) / 2} .
$$

Proof. As a matter of fact, if $g \in H_{(\lambda-1) / 2}^{\infty}$, then for any $f \in \mathcal{L}_{0}^{2, \lambda}$, $I_{g} f \in B_{0}$. Since $f_{n}(z) \in \mathcal{L}_{0}^{2, \lambda}$ (see Theorem 4.4), we complete the proof as the same as in the proof of Theorem 4.4. 
Theorem 5.3. Suppose that $0<\lambda<1$ and $g \in H(\mathbb{D})$. Then the following conditions are equivalent:

(i) $T_{g}: \mathcal{L}_{0}^{2, \lambda} \rightarrow B_{0}$ is bounded;

(ii) $g \in B \mathcal{L}_{0}^{p, \lambda}$ for all $p \in(1, \infty)$;

(iii) $g \in B \mathcal{L}_{0}^{p, \lambda}$ for some $p \in(1, \infty)$.

Moreover,

$$
\left\|T_{g}\right\| \approx M(g)
$$

Proof. (i) $\Rightarrow$ (ii). Suppose that $T_{g}$ is bounded. For any $I \subset \partial \mathbb{D}$, let $b=(1-|I|) \zeta$, where $\zeta$ is the center of $I$. Then

$$
\left(1-|b|^{2}\right) \approx|1-\bar{b} z| \approx|I|, z \in S(I) .
$$

Concerning the functions $F_{b}(z)$ in Lemma 3.1, we have $F_{b}(z) \in \mathcal{L}_{0}^{2, \lambda}(\mathbb{D})$; thus, $T_{g}\left(F_{b}(z)\right) \in B_{0}$. Furthermore, from Proposition 2.2, it yields $g \in B \mathcal{L}_{0}^{p, \lambda}$.

(ii) $\Rightarrow$ (iii). It is obvious.

(iii) $\Rightarrow\left(\right.$ i). Fix $p \in(1, \infty)$ and $g \in B \mathcal{L}_{0}^{p, \lambda}$. Then from Theorem 3.4, $T_{g}: \mathcal{L}^{2, \lambda} \rightarrow B$ is bounded, and hence $T_{g}: \mathcal{L}_{0}^{2, \lambda} \rightarrow B$ is bounded. It suffices to prove that, for any $f \in \mathcal{L}_{0}^{2, \lambda}, T_{g} f \in B_{0}$. Indeed, $g \in B \mathcal{L}_{0}^{p, \lambda}$, for every $\varepsilon>0$ there is a constant $\delta>0$ such that, as $|J|<\delta$,

$$
\frac{1}{|J|^{p-\lambda+1}} \int_{S(J)}\left|g^{\prime}(z)\right|^{2}\left(1-|z|^{2}\right)^{p} d m(z)<\varepsilon .
$$

With the above $\delta$, for any $|I|<\delta$, we break up $S(I)$ in the same way as in Theorem 3.4. Then, by (3.2),

$$
\begin{aligned}
& \int_{S(I)}\left|g^{\prime}(z)\right|^{2}\left(1-|z|^{2}\right)^{p+\lambda-1} d m(z) \\
& \lesssim \sum_{n=0}^{\infty} \sum_{J \in \mathcal{D}_{n}(I)} \int_{S(J)}|J|^{\lambda-1}\left|g^{\prime}(z)\right|^{2}\left(1-|z|^{2}\right)^{p} d m(z) \\
& \leq \sum_{n=0}^{\infty} \sum_{J \in \mathcal{D}_{n}(I)} \varepsilon|J|^{\lambda-1}|J|^{p-\lambda+1} \\
& \lesssim \varepsilon|I|^{p}
\end{aligned}
$$


namely,

$$
\lim _{|I| \rightarrow 0} \frac{1}{|I|^{p}} \int_{S(I)}\left|g^{\prime}(z)\right|^{2}\left(1-|z|^{2}\right)^{p+\lambda-1} d m(z)=0 .
$$

Now, it is easy to see that

$$
\begin{aligned}
& \lim _{|I| \rightarrow 0}\left(\frac{1}{|I|^{p}} \int_{S(I)}|f(z)|^{2}\left|g^{\prime}(z)\right|^{2}\left(1-|z|^{2}\right)^{p} d m(z)\right)^{1 / 2} \\
& \lesssim\|f\|_{\mathcal{L}^{2, \lambda}} \cdot \lim _{|I| \rightarrow 0}\left(\frac{1}{|I|^{p}} \int_{S(I)}\left|g^{\prime}(z)\right|^{2}\left(1-|z|^{2}\right)^{p+\lambda-1} d m(z)\right)^{1 / 2}=0 .
\end{aligned}
$$

In conclusion, $T_{g}: \mathcal{L}_{0}^{2, \lambda} \rightarrow B_{0}$ is bounded.

Lemma 5.4. Suppose that $0<\lambda<1,1<p$ and $g \in B \mathcal{L}_{0}^{p, \lambda}$ and the operator $T_{g, r}: \mathcal{L}_{0}^{2, \lambda} \rightarrow B_{0}$ satisfies

$$
T_{g, r} f(z)=\int_{0}^{z} f(r w) g^{\prime}(w) d w
$$

where $r \in(0,1)$. Then $T_{g, r}: \mathcal{L}_{0}^{2, \lambda} \rightarrow B_{0}$ is compact.

Proof. Since $g \in B \mathcal{L}_{0}^{p, \lambda}$, it follows from Lemma 4.3 that $T_{g, r}: \mathcal{L}^{2, \lambda} \rightarrow$ $B$ is compact, and hence $T_{g, r}: \mathcal{L}_{0}^{2, \lambda} \rightarrow B$ is compact. As a matter of fact, in Theorem 5.3, if $g \in B \mathcal{L}_{0}^{p, \lambda}$, then for any $f \in \mathcal{L}_{0}^{2, \lambda}, T_{g} f \in B_{0}$. Together with Lemma 4.1, we conclude $f \in \mathcal{L}_{0}^{2, \lambda}, T_{g, r} f \in B_{0}$, so that $T_{g, r}: \mathcal{L}_{0}^{2, \lambda} \rightarrow B_{0}$ is compact.

Theorem 5.5. Let $0<\lambda<1$ and $g \in H(\mathbb{D})$. If $T_{g}: \mathcal{L}_{0}^{2, \lambda} \rightarrow B_{0}$ is bounded, then

$$
\left\|T_{g}\right\|_{e, \mathcal{L}_{0}^{2, \lambda} \rightarrow B_{0}} \approx \limsup _{|a| \rightarrow 1}\left(\int_{\mathbb{D}}\left(\frac{1-|a|^{2}}{|1-\bar{a} z|^{2}}\right)^{p+1-\lambda}\left|g^{\prime}(z)\right|^{2}\left(1-|z|^{2}\right)^{p} d m(z)\right)^{1 / 2} .
$$

Proof. Noting that $F_{n}(z) \in \mathcal{L}_{0}^{2, \lambda}$ and the compact operator $T_{g, r}$ in Lemma 5.4, we can obviously complete the proof as the same as in proof of Theorem 4.2. 
The following corollary is an immediate consequence of the above theorem.

Corollary 5.6. Let $0<\lambda<1$ and $p>1$. Then

(i) $I_{g}: \mathcal{L}_{0}^{2, \lambda} \rightarrow B_{0}$ is compact if and only if $g=0$.

(ii) $T_{g}: \mathcal{L}_{0}^{2, \lambda} \rightarrow B_{0}$ is compact if and only if $T_{g}: \mathcal{L}_{0}^{2, \lambda} \rightarrow B_{0}$ is bounded if and only if $g \in B \mathcal{L}_{0}^{p, \lambda}$.

\section{REFERENCES}

1. D. Adams and J. Xiao, Nonlinear potential analysis on Morrey spaces and their capacities, Indiana Univ. Math. J. 53 (2004), 1629-1663.

2. _ Morrey spaces in harmonic analysis, Ark. Mat. 50 (2012), 201-230.

3. A. Aleman and J.A. Cima, An integral operator on $H^{p}$ and Hardy's inequality, J. Anal. Math. 85 (2001), 157-176.

4. A. Aleman and A.G. Siskakis, Integration operators on Bergman spaces, Indiana Univ. Math. J. 46 (1997), 337-356.

5. J.M. Anderson, Bloch functions: The basic theory, operators and function theory, Math. Phys. Sci. 153 (1985), 1-17.

6. J.M. Anderson, J. Clunie and Ch. Pommerenke, On Bloch functions and normal functions, J. reine angew. Math. 270 (1974), 12-37.

7. R. Aulaskari and P. Lappan, Criteria for an analytic function to be Bloch and a harmonic or meromorphic function to be normal, in Complex analysis and its applications, Pitman Res. Not. Math. 305 (1994), 136-146.

8. R. Aulaskari, D.A. Stegenga and J. Xiao, Some subclasses of BMOA and their characterizations in terms of Carleson measures, Rocky. Mountain J. Math. 26 (1996), 485-506.

9. K.D. Bierstedt, J. Bonet and A. Galbis, Weighted spaces of holomorphic functions on bounded domains, Michigan Math. J. 40 (1993), 271-297.

10. K.D. Bierstedt and W.H. Summers, Biduals of weighted Banach spaces of analytic functions, J. Austr. Math. Soc. 54 (1993), 70-79.

11. C. Cascante, J. Fàbrega and J.M. Ortega, The Corona theorem in weighted Hardy and Morrey spaces, Ann. Sc. Norm. Super. Pisa, DOI 10.2422/2036-2145.201202 006.

12. O. Constantin, A Volterra-type integration operator on Fock spaces, Proc. Amer. Math. Soc. 140 (2012), 4247-4257.

13. C. Cowen and D. MacCluer, Composition operators on spaces of analytic functions, Stud. Adv. Math., CRC Press, Boca Raton, FL, 1995.

14. P. Duren, Theory of $H^{p}$ paces, Academic Press, New York, (1970).

15. D. Girela, Analytic functions of bounded mean oscillation, Complex function spaces, Univ. Joensuu Dept. Rep. Ser. 4, University of Joensuu, Joensuu, 2001. 
16. J. Laitila, S. Miihkinen and P. Nieminen, Essential norms and weak compactness of integration operators, Arch. Math. 97 (2011), 39-48.

17. P. Li, J. Liu and Z. Lou, Integral operators on analytic Morrey spaces, Sci. China Math. 57 (2014), 1961-1974.

18. J. Liu, Z. Lou and C. Xiong, Essential norms of integral operators on spaces of analytic functions, Nonlin. Anal. 75 (2012), 5145-5156.

19. S. Li and S. Stević, Volterra-type operators on Zygmund spaces, J. Inequality Appl. Article ID 32124, (2007), 10 pages.

20. K. Madigan and A. Matheson, Compact composition operators on the Bloch space, Trans. Amer. Math. Soc. 347 (1995), 2679-2687.

21. C.B. Morrey, Jr., On the solutions of quasi-linear elliptic partial differential equations, Trans. Amer. Math. Soc. 43 (1938), 126-166.

22. J. Peetre, On the theory of $\mathcal{L}_{p, \lambda}$ spaces, J. Funct. Anal. 4 (1964), 71-87.

23. Ch. Pommerenke, Schlichte Funktionen und analytische Funktionen von beschränkter mittlerer Oszillation, Comment. Math. Helv. 52 (1977), 591-602.

24. J. Shapiro, The essential norm of a composition operator, Ann. Math. 125 (1987), 375-404.

25. A.L. Shields and D.L. Williams, Bounded projections, duality, and multipliers in spaces of harmonic functions, J. reine angew. Math. 299 (1978), 256-279.

26. , Bounded projections and the growth of harmonic conjugates in the disk, Michigan Math. J. 29 (1982), 3-25.

27. A. Siskakis and R. Zhao, A Volterra type operator on spaces of analytic functions, Contemp. Math. 232 (1999), 299-311.

28. J. Wang and J. Xiao, Holomorphic Campanato spaces on the unit ball, arXiv:1405.6192v1 [math.CV].

29. , Two predualities and three operators over analytic Campanato spaces, arXiv: $1402.4377 \mathrm{v} 1$ [math.CV].

30. Z. Wu, A new characterization for Carleson measure and some applications, Int. Equat. Oper. Theor. 71 (2011), 161-180.

31. Z. Wu and C. Xie, Q spaces and Morrey spaces, J. Funct. Anal. 297 (2003), $282-297$.

32. H. Wulan and J. Zhou, $Q_{K}$ and Morrey type spaces, Ann Acad. Sci. Fenn. Math. 38 (2013), 193-207.

33. J. Xiao, The $Q_{p}$ Carleson measure problem, Adv. Math. 217 (2008), 20752088.

34. Holomorphic Q classes, Lect. Notes Math. 1767 (2001), SpringerVerlag, Berlin.

35. , Geometric $Q_{p}$ functions, Frontiers in Mathematics, Birkhäuser Verlag, Basel, 2006.

36. J. Xiao and $\mathrm{W}$. Xu, Composition operators between analytic Campanato spaces, J. Geom. Anal, doi 10.1007/s12220-012-9349-6. 
37. J. Xiao and C. Yuan, Analytic Campanato spaces and their compositions, arXiv: $1303.5032 \mathrm{v} 2$ [math.CV].

38. S. Ye, Norm and essential norm of composition followed by differentiation from logarithmic Bloch spaces to $H_{\mu}^{\infty}$, Abst. Appl. Anal. Art. ID 725145, (2014), 6 pages.

39. Products of Volterra-type operators and composition operators on logarithmic Bloch space, WSEAS Trans. Math. 12 (2013), 180-188.

40. S. Ye and J. Gao, Extended Cesáro operators between different weighted Bloch-type spaces, Acta Math. Sci. 28 (2008), 349-358 (in Chinese).

41. K. Zhu, Operator theorey in function spaces, Second edition, Math. Surv. Mono. 138 (2007).

42. C.T. Zorko, Morrey space, Proc. Amer. Math. Soc. 98 (1986), 586-592.

Department of Mathematics, Shantou University, Shantou 515063, China and Department of Mathematics, Fujian Normal University, Fuzhou 350007, CHINA

Email address: 14zyzhuo@stu.edu.cn

School of Sciences, Zhejiang University of Science and Technology, Hangzhou 310023, China and Department of Mathematics, Fujian Normal University, Fuzhou 350007, China

Email address: shanliye@fjnu.edu.cn, ye_shanli@aliyun.com 